\title{
Let's Join: A Pavilion Inspired by the Weaire and Phelan Space Tessellation
}

\section{Graziano Mario Valenti $^{1}$ (D) Chadi El Khoury $^{1}$}

Accepted: 27 December 2020 / Published online: 13 January 2021

(C) The Author(s) 2021

\begin{abstract}
The pavilion presented in this paper is an articulation of space based on the Weaire and Phelan tessellation. In this pavilion a second generative pattern is partially engraved and cut out of the polygonal surfaces that bind the polyhedron. This pattern is made up of cubic curves that aim to spatially engage with the tessellation. The design and prototyping process was implemented and controlled using parametric and procedural models. The use of these models made it possible to define the shape, orientation, and size of each element of the pavilion: from the polyhedron and the pattern's curves, to smaller components like the shape of the panels' joints, holes, and the countersinks that hold the screws. This research illustrates the geometric, formal, and procedural design that was used to shape the pavilion in its current spatial configuration, to draw the pattern cut and engraved into the polyhedron's surface, and finally, to determine the shape and scale of the joints that hold the faces of the polyhedron together and characterize the internal space. Regarding the topic of Patterns and Spatial Organization, the paper investigates shape design methodologies, which are based on historical practices but updated using new technologies.
\end{abstract}

\section{Introduction}

Let's Join is a small modular and expandable pavilion, built in September 2019 at the Debbye campus of the Beirut Arab University in Lebanon. The pavilion was designed in the context of an international cooperation project between the Sapienza University of Rome and the Beirut Arab University. Both institutions were directly involved in handling its realization (Fig. 1). The name Let's Join, conceptualized

Graziano Mario Valenti

grazianomario.valenti@uniroma1.it

Chadi El Khoury

s.khoury@bau.edu.lb

1 Dipartimento di Storia Disegno e Restauro Dell'Architettura, Universita Degli Studi di Roma La Sapienza, Rome, Italy

Birkhäuser 

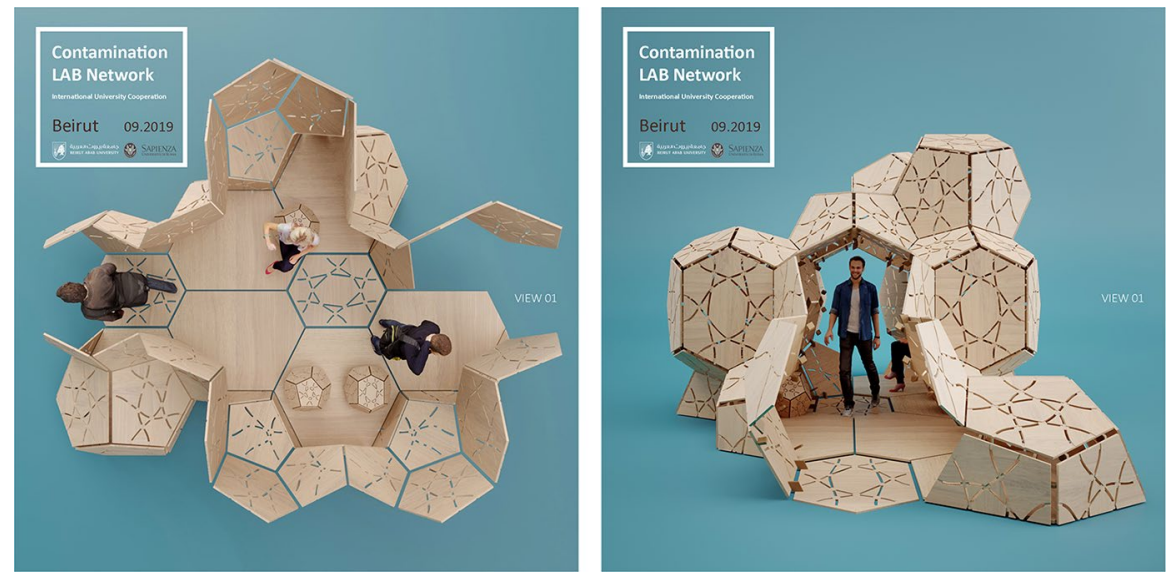

Fig. 1 The "Let's Join" pavilion

during the process, has multiple meanings: it represents the scientific collaboration between the two universities; it expresses the geometric properties of the structure; and lastly it describes its purpose as a space for the BAU students to meet and socialize. The international cooperation aims to define an operational protocol for training and experimentation, to be used by manufacturing laboratories. Its main focus is geometric theory and its application to the design and construction of the architectural form and, more generally, of the industrial product.

\section{"Ex ante", "in itinere" and "ex post" Digital Processing}

Historically, knowledge of geometry was a fundamental part of the "ex ante" phase of design. The quality and variety of the designer's geometric understanding was therefore the basis of the origin and means of expression of the form. In the works of Pier Luigi Nervi, Felix Candela and Santiago Calatrava, for example, it is clear that the aesthetic of form was the origin of the design idea and was produced by the synthesis of the geometry-form-structure relationship. Less frequent, however, was the intervention of geometry in the "in itinere" phase of design. This term is relevant, for example, to Otto Frei's work on minimal surfaces. Throughout his process, geometry, although created experimentally by weaving soap films on iron looms, was nevertheless deeply understood by the designer, thanks to his experience and theoretical foundations.

Additionally, geometry can also pervade into the "ex post" phase, where it intervenes after a free form has already been defined. Here, geometry relates to the work in an intuitive-perceptive way, working with the approach of a sculptor. These shapes must be rationalized to satisfy constructional limits during a subsequent phase, which focuses on the optimization of costs and construction processes. An approach of this type can be found in some of the architectural works of Frank O. Gehry or Massimiliano Fuksas, where the shape optimization process reduces 
the external envelope - created freely and without geometric thought - to sections of developable surfaces or generic polyhedral faces. The sudden developments of digital technologies that make this process possible encourage new generations of designers to work primarily "ex post," rarely "in itinere" and even more rarely "ex ante". Consider, for example, the innovation of recent generative modelling aids like Autodesk Generative Design: with these tools, the process of determining the shape in its optimized structure, is completely entrusted to digital processing. If, on one hand, these technologies offer extraordinary opportunities in terms of identifying ideal forms, on the other they cause considerable concerns about the aesthetic of their results, the identity of the forms generated by automatic processes, and the designer's reduced ability to intervene in the creation of an optimized form.

These introductory reflections, therefore, aim to refocus attention and debate on the geometric reasoning behind form, which constitutes a central aspect of the development of various research fields; at the same time, they seek to motivate new generations of designers to preserve the historical memory and the practice of operating in compliance with the cultural contents and ethical principles of the project that emerged from geometry and characterized all pre-digital eras. This was a remarkably significant period, which cannot be forgotten, where the complexity of form was created by an artistic process arising from a deep knowledge of geometry and structure. This complexity could never be generated, as today appears possible, with a simple gestural act. The collaboration between Universities in this project was designed to explore the geometric shape and its peculiar qualities.

\section{Why a Wooden Pavilion?}

Wishing to work with models at the scale of the architectural space, but at the same time having the need to identify a prototype subject that could be repeated in other experimental applications, the design choice fell on the theme of the wooden pavilion. The pavilion presented a unique architectural typology that, if necessary, could have multiple dimensions and functions, and that could be achieved with navigable difficulties in other university fabrication laboratories, with heterogeneous operational dimensions, equipment and budgets. The subject, which for the reasons indicated has been frequently investigated in the university research, brings to the forefront themes such as geometric and constructive modularity, robotic manufacturing, structural efficiency, and behaviour with respect to environmental phenomena, to name a few examples. Among the most significant research experiences, those produced at the University of Stuttgart, stemming from the collaboration between the Institute for Computational Design (ICD) and the Institute of Building Structures and Structural Design (ITKE), must certainly be included. The monumental construction, characterized by a strong interdisciplinary collaboration perfected over a long period of time, consists of several wooden pavilions. In the Institutes' projects the geometric quality of the shape is inspired by the natural world and perfected through a path of careful observation and subsequent conceptual synthesis. This biomimicry activity is crucial to arrive at formal and constructive innovative solutions. From a strictly geometric point of view, the 
adaptive tessellation criteria of surfaces and the three spatial interpretations of the related modules in the form of flat surfaces (Landesgartenschau Exhibition Hall of 2014) (Krieg et al. 2015), coffered panels (Research Pavilions of 2011 and Buga Pavilion of 2019) (Schwinn et al. 2012; Alvarez et al. 2019) and single curvature (Research Pavilions of 2015-16) (Schwinn et al. 2016) are particularly interesting. An additional Hygroskin, a Meteorosensitive Pavilion, was created at the University of Stuttgart, by a research group led by Archim Memges and with the help of the ICD, (Krieg et al. 2013). In this project, the responsive surfaces that autonomously open and close in response to weather changes are investigated: a project that uses the responsive capacity of the material itself. This experience is mentioned because Let's Join, of which only the "motionless" structural part is illustrated here, was created with the objective of hosting experimental prototypes of responsive surfaces, including both mechanical activation and natural behaviour, within the empty pentagonal modules.

Also inspired by biomimicry is the Bowooss Bionic pavilion, (Pohl 2001) built at Saarland University's School of Architecture. In this project, structural concepts relating to joints and the relationship between solids and voids, characteristic of diatoms, were applied to a polyhedral surface made following the miura-ori pattern. The "Pavilion for a Summer" (Hartmann et al. 2013), designed in 2013 by Manuel Fabian Hartmann and his team in the "Design and Construction" course at the University of Innsbruck, is smaller and easier to build. Hartmann used a Voronoi tessellation, optimized with respect to the structural behaviour. The modules are made in the form of surfaces with single curvature. Experimentation on wooden pavilions takes on a slightly different approach in the joint research of the EmTech Program (AA School, London) and the DARCH Chair of Structural Design at ETH Zurich (D'Acunto and Kotnik 2013). This research explored the relationship between form and structural resistance, creating a vault composed of three thin layers of plywood cut to increase bending and connected to each other with steel cables. In another research project, the Italian National Research Council built the FlexMaps Pavilion (Malomo et al. 2018; Laccone et al. 2019), a $4 \times 4 \mathrm{~m}$ structure made of CNC-milled plywood. A computationally designed spiralling pattern was carved into the structure. This pattern aimed at controlling the flexibility of each panel so that, once all the pieces were individually bent and assembled, the structure would reach its target shape.

\section{The Choice of the Weaire and Phelan Tessellation}

The main requirement for the Let's Join pavilion was that it needed to be reproducible in different shapes and sizes, by simply increasing and freely modifying the modules of a spatial pattern. Therefore, the research focused on the analysis of the geometric forms helpful for tessellating space. The tessellation of Euclidean space can be implemented in a regular form only using the cube, and in a semiregular form either with a combination of regular solids or with a combination of regular Platonic solids and semiremiregular Archimedes solids (Baglioni 2009). Of course, there are infinite ways to tessellate space using irregular solids. After a survey and some simulations 
on regular structures, the appeal of minimal surfaces led the architects to choose Weaire's and Phelan's tessellation. In addition to optimizing material with respect to its volpublisume, the tessellation is easy to configure so that it perceptively produces a non-monotonous articulation. This type of tessellation is the one that best solves Lord Kevin's problem, who in 1887 wondered how to tessellate a space using the least number of equal-volume cells and minimum contact surface. As it is well-known, Kevin found the solution in a structure with truncated octahedrons, Archimedean polyhedra of 14 faces, of which six are squares and eight are hexagons. In 1993, Denis Weaire and Robert Phelan, with the aid of digital simulation, created a structure with a surface area that was $0.3 \%$ smaller than the one proposed by Kevin (Weaire 1996). The tessellation developed by Weaire and Phelan is made with two different types of cells. The first is a pentagonal dodecahedron cell with irregular faces (pyritohedron). The second is a truncated hexagonal trapezohedron, i.e. an irregular tetrakaidecahedron having two hexagonal faces and 12 pentagonal faces.

The tessellation of Weaire and Phelean is frequently explored and experimented with in the digital form in the designs of buildings, exhibition spaces, artistic works, and industrial objects, but it is very rarely found in projects that reach the construction stage. Noteworthy are two works that effectively represent the advantages and aesthetic value of this particular tessellation of space: The Water Cube Aquatics Center in Beijing, designed by architects Chris Bosse, Rob and Leslie-Carter for the Beijing Olympic Games of 2008 (Pohl 2008), and the floating sculpture On the Disappearance of Clouds, created by the Argentine artist Tomás Saraceno for the 2019 Venice Biennale (Malone and Marjanovic 2014). Both creations benefited from the direct collaboration of Denis Weaire. In Beijing's Water Cube Aquatics Center, the tessellation seems enclosed in a right prism with a rectangular base whose surfaces dissect the polyhedra's pattern. A $60^{\circ}$ rotation applied to the pattern produces a variety of sections of the polyhedra, which deceives the visitor, who thus has the impression of facing a random pattern. In the work, On the Disappearance of Clouds, the polyhedra of the tessellation are suspended in the air in small groups by means of steel cables and are represented through their edges and only some of their faces. In both cases, the spatiality of the polyhedral articulation is perceivable only from a distance and from the outside, but it cannot be appreciated from the inside. Inside the Watercube, in fact, the visitor is struck by the spatial void of the prism that contains the tessellation rather than by the tessellation itself, which affects only the boundary surfaces of the architecture. In Saraceno's work, internal use of the structure is not even possible. Thus, the creation of the Let's Join pavilion was particularly interesting because it provided an opportunity to experience and confront, on an anthropomorphic scale, the spatial tessellation of Weaire and Phelan.

\section{Articulation of the Polyhedral Pattern}

Because the Weaire and Phelan tessellation is made from minimal surfaces, the pentagonal and hexagonal faces are slightly curved in the theoretical model. The representation with polyhedra is, therefore, an approximation that rectifies them by 


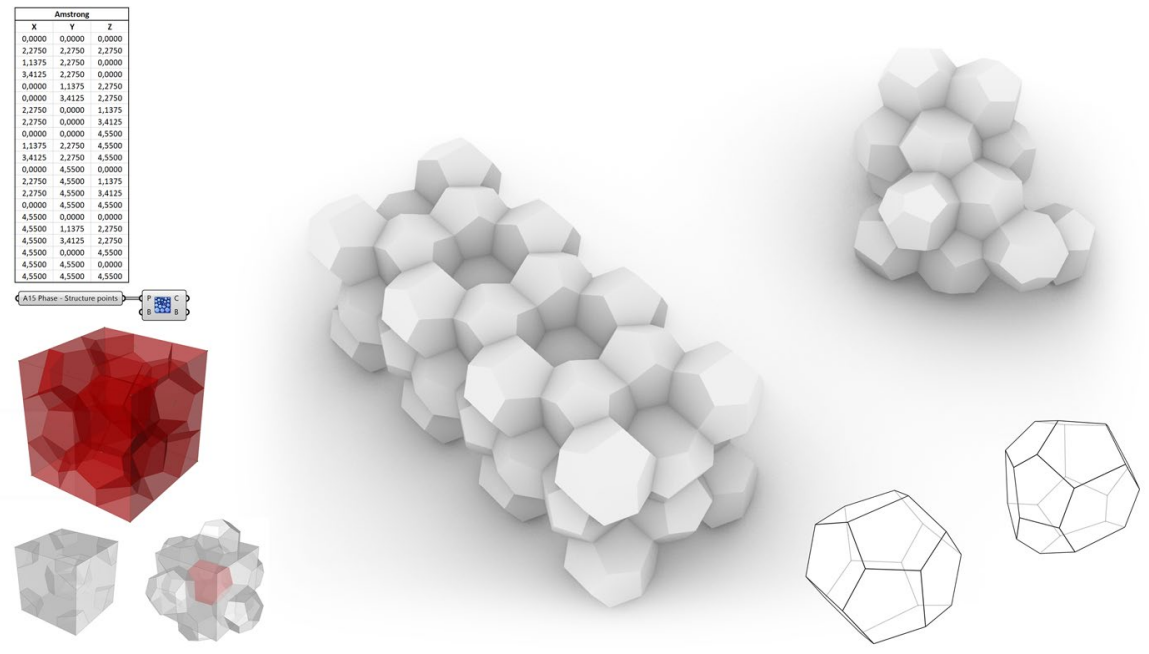

Fig. 2 Weaire and Phelan tessellation: 3d modelling and selection

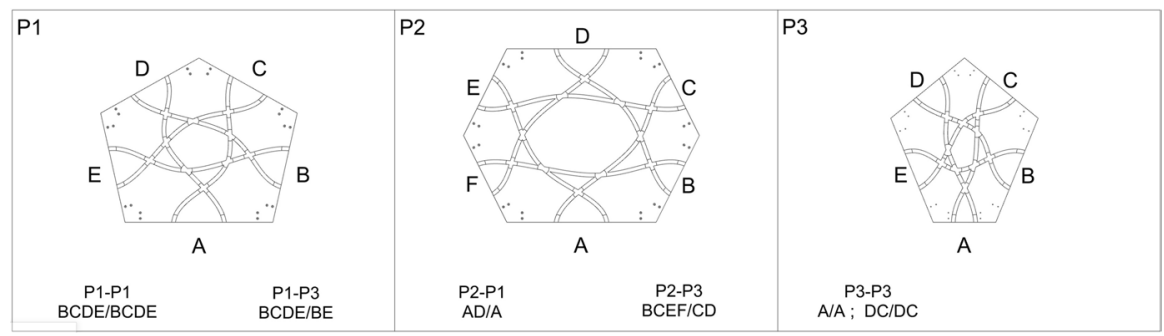

Fig. 3 The polyhedron of the pavilion has only three types of panels. These could be easily assembled reading the connection code (e.g.: side B, C, D, E of a panel P1 could be joined with the sides B and E of a panel P3)

flattening their faces. The tessellation of Weaire and Phelan can be reproduced-in an approximate form - by applying the Voronoi algorithm to a series of points in a space distributed according to the A15 Phase crystallographic structure defined by Frank and Kasper in 1958.

At first, the main module of the pattern was created, completing the constituent polyhedra with mirror copies. The primary module was then replicated to have a number of polyhedra on which to apply a selective action that defined the pavilion's space (Fig. 2). These first operations were carried out by transforming the coordinates of the crystallographic structure into the architectural scale, equating 1 Amstrong $=1 \mathrm{Mt}$.

After the shape of the two polyhedral was created, the maximum measurements of the polygonal faces of the polyhedral were examined. The polyhedrons of the pattern have three types of irregular polygonal faces: two are pentagonal and one is hexagonal (Fig. 3). 
Once the maximum dimensions normally achievable in prototyping were assessed, the pattern was scaled so that each element could fit in the material used (wood) and within the operating limits of the prototyping equipment (cutter). In particular, given the plan to create the wooden pavilion and then replicate it in other workshops, the standard sizes of table and cutter were chosen, focusing on the $240 \times 120$ format, which generally has a few additional $\mathrm{cm}$. of margin. Moreover, considering different possibilities for nesting the polygonal faces, the dimensional constraint was identified in the height of the largest pentagon, which could at most be $90 \mathrm{~cm}$. Functional selective operations were performed on the polyhedrons used for the tessellation to ensure access and usability of the internal space. First, ground 0 was defined by sectioning the pattern with a horizontal plane in correspondence with the hexagonal face of the tetracaidecaèdro. This sectioning determined the shape of the design of the pavilion floor, which only in some modules coincides and formally refer to the face of the tetracaidecaèdro. Afterward, the minimum number of modules necessary to configure a usable space for a brief visit of 3 or 4 people was identified. Finally, some faces of the modules were removed to provide access to the space delimited by the pattern and to subsequently introduce panels with responsive surfaces (Fig. 4).

\section{From the design of the geometric model to the design of the physical model}

One of the goals of the project was to make the modules and their faces easy to assemble with core procedures suitable for students of future workshops who may have minimal wood processing and assembly skills. To meet this requirement, it was decided to connect the modules' faces in only two points on each side through the
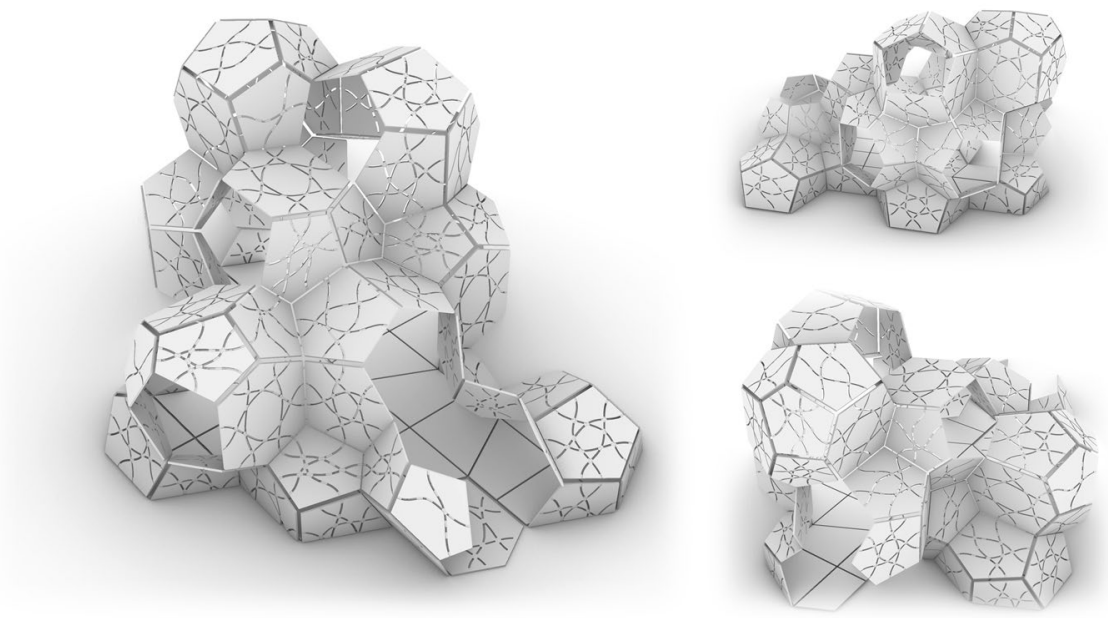

Fig. 4 The final articulation of the pavilion 
use of simple joints that also play the role of spacers. In this way, the adjacent sides are distinct, and an evocative line of light draws the articulation of the polyhedron, from the inside during the day and from the outside during the night. The threedimensional creation of the pattern's panels followed this formal development: at the polygons delimiting each face of the polyhedron a $20 \mathrm{~mm}$ offset was applied towards the inside. The resulting polygons were extruded $18 \mathrm{~mm}$ (thickness of the wooden boards) towards the outside of the polyhedron space (Fig. 5). In this way, the shape, orientation, and position of every single panel of the polyhedron forming the pavilion was obtained. The actual configuration is made of 24 panels of type $\mathrm{P} 1,9$ of type P2, and 33 of type P3. There are also ten panels of type P1b, and four of type P2b, necessary to establish the connection of the pavilion to the ground, where the horizontal plane sectioned the pattern. Panels P1b and P2b correspond, respectively, to half of the panels $\mathrm{P} 1$ and $\mathrm{P} 2$. The section of the horizontal plane also generated two types of modules that draw the floor surface. Because the modules of the floor are larger than the ones achievable with the prototyping equipment, they were further divided into more parts. Occasionally, the hexagonal module corresponding to the face of the tetracaidecaèdro crossed by the section plane occurs within the pattern (Fig. 5).

\section{The Cubic Curves Pattern}

The pavilion's interior is strongly characterized by the presence light and shadow. To develop the space, a solution was adopted that on one hand dematerialized the ideal edges of the polyhedron attributing its representation to the light. On the other hand, it led to creating a texture of intersecting incisions and cuts in the form of cubic curves, generated by an automatic semi-random procedure on

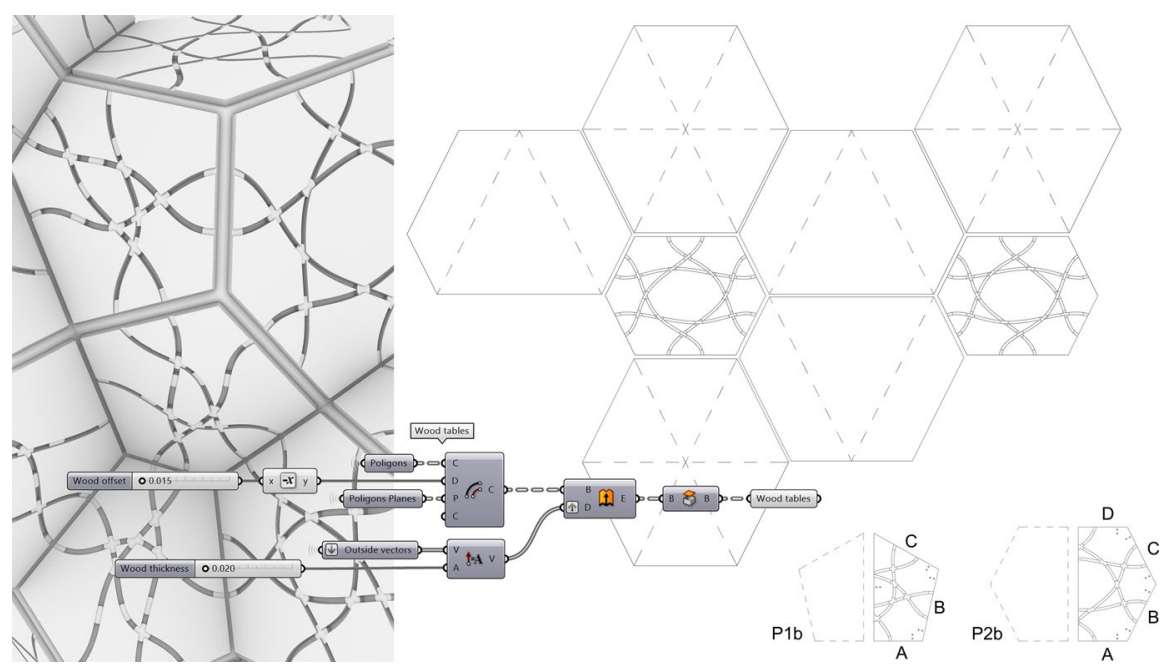

Fig. 5 The extrusion of the panels (left); the pavilion floor and the subpanels P1b and P2b (right) 
the polyhedron's faces. This texture is not only decorative, but it also lightens the panels without weakening them. Further, it allows light to penetrate and illuminate the space. This light interacts with shadows to draw geometric patterns on the floor and the interior surfaces of the polyhedron. The geometric structure of the plan represents a union between the free form and the geometry-based form. The first is expressed, through approximation (Nurbs), by a pattern of cubic curves, the second is represented by the multifaceted surface of Weaire and Phelan structure. Initially, the described pattern appears to move with "freedom" on the surface of the polyhedron, but after closer inspection, its mathematical nature and geometric constraints emerge. The aim of the design of this second pattern is to produce curves that are distributed on the surface of the polyhedron without discontinuity. Additionally, the curves must be aesthetically variable, and at the same time leave space for the connection of the joints and be placed near the sides of the polygon with the tangent perpendicular to them. The following procedural rules were adopted (Fig. 6).

1. The curve is a Nurbs of the 3rd degree made with five control points, and is therefore composed of two cubic arcs

2. Two points are placed on each side of the polygon so that they are equally spaced out between themselves and the vertices

3. The curve always starts from the first of the two points and ends at the second of the two points on the side adjacent to the origin side. This process is carried out counter clockwise

4. The first and second points as well as the fourth and fifth points of the Nurbs control polygon-which define the tangent of the curve at both its start and ending points - are positioned on straight lines perpendicular to the sides of the polygon
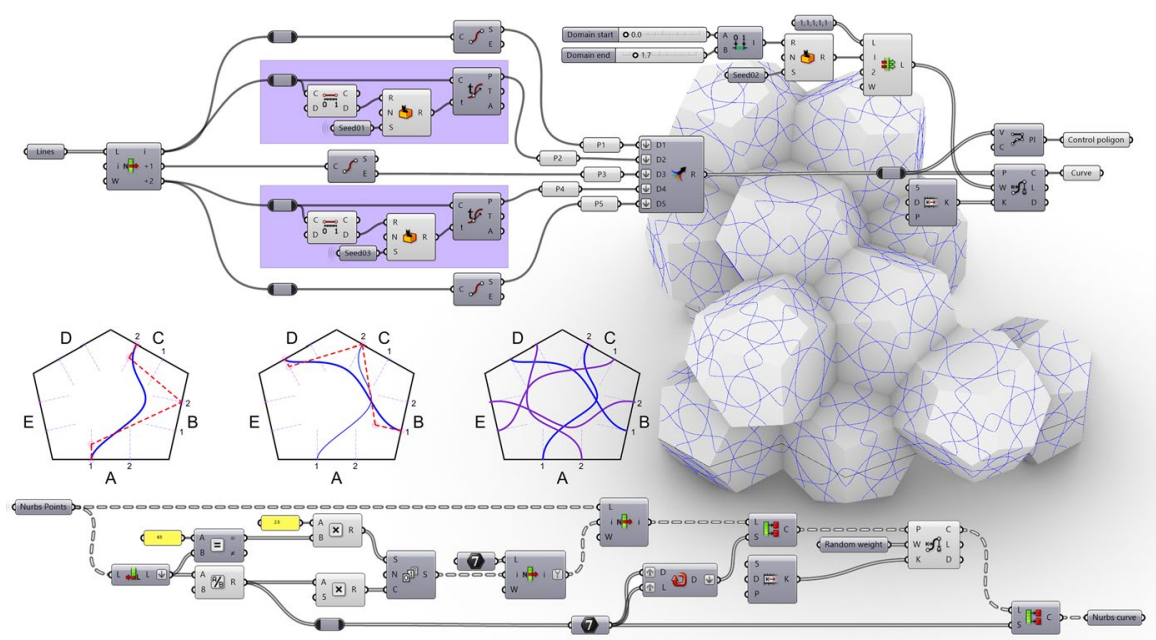

Fig. 6 The procedural genesis of the cubic curves pattern 
5. The distance between these points is calculated randomly within an assigned domain, introducing the first arbitrary variation of the curve. This variation affects how quickly it expresses its curvature

6. The third point of the curve is always located at the second point of the first side adjacent to the origin side of the curve

7. Each control point of the Nurbs curve has a weight of 1, with the exception of the third, which has a randomly determined weight within an assigned domain. Thus, this creates the second arbitrary variation of the curve, affecting how close it is to the side of the polygon.

The pattern's curves - six on the hexagon and five on the pentagon-repeatedly intersect each other and completely cut through the panel in their path. It is therefore impossible to perform full cuts along the curves without fragmenting the panel into several pieces. To overcome this problem, a cylindrical volume was placed each time the curves intersected each other and the edge of the panel. Within this volume the cut was replaced with a simple incision.

\section{Joints of the Panels}

As previously described, simple joints were created to assemble the wooden panels making up the surfaces of the polyhedron. These joints simultaneously acted as spacers, leaving a "line of air" between one panel and the other. Thus, the architects avoided the use of more complex joining solutions, which would have been necessary to connect boards of homogeneous thicknesses at different angles. The line of air, moreover, invisibly absorbs small assembly inaccuracies and necessary adjustments. The panels, spaced in this way, seem to float in space, which positively contributes to the aesthetic impact of the pavilion. The joints were made with wood of the same type and thickness as the panels. They were built as trapezoids, and oriented so that their parallel sides were also parallel to the panels they were joined to. The spacer corresponds with one of trapezium's vertices. Each spacer has two planes where the panel rests and two small cylinders that allow for the correct movement of the tip of the milling machine $(\varnothing 12 \mathrm{~mm})$. The pattern requires a total of seven types of joints, which become 14 if duplicated considering that the convergence of the panels is sometimes concave and sometimes convex. To these, the exceptions where the pattern touches the section plane on the ground must be added. To secure all the panels of the pavilion a total of 364 joints were produced. Each pair of panels sharing a side is connected with two joints. The joints are placed near the vertices of the sides of the panel at a fixed distance of $15 \mathrm{~cm}$. Therefore, each vertex of the polyhedron has three joints that are close to each other. When the joints are combined, the user views them as a connecting hexahedron. Each joint is scaled so that it can fit a pair of $7 \mathrm{~cm}$ screws that are positioned $3 \mathrm{~cm}$ and $5 \mathrm{~cm}$ from the edge of the panel. The joints were also drawn automatically through a procedural parametric algorithm. Held together in this way, the structure is self-supporting (Fig. 7). 


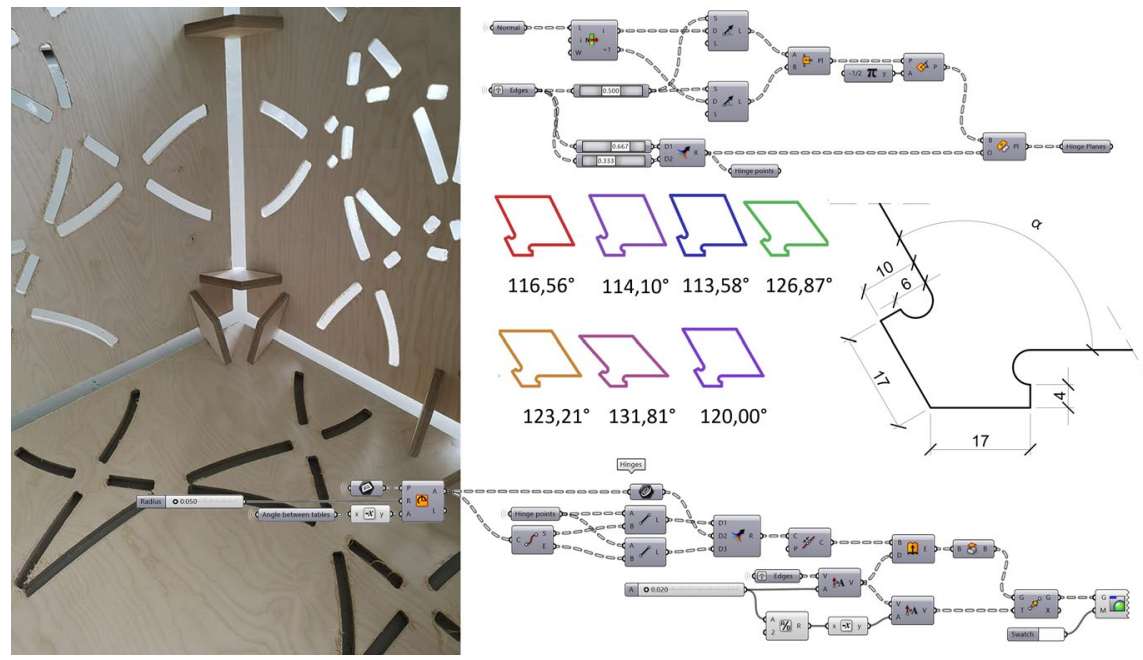

Fig. 7 Their combination of three joints is perceived by the user as a connecting hexahedron (left). Design and procedural algorithm of the joints (right)

\section{Prototyping and Assembly}

The prototyping was carried out primarily using a three-axis CNC milling machine. This tool cut out the panels, performed incisions within the panels, and finally, drilled countersinks and holes into them. Because of the little variety of panel types, it was not necessary to use special nesting procedures that optimized the use of the wooden boards (Fig. 8). Only five table formats were produced and later sent to the cutter to obtain all the necessary panels. A total of 22 wooden boards were used to
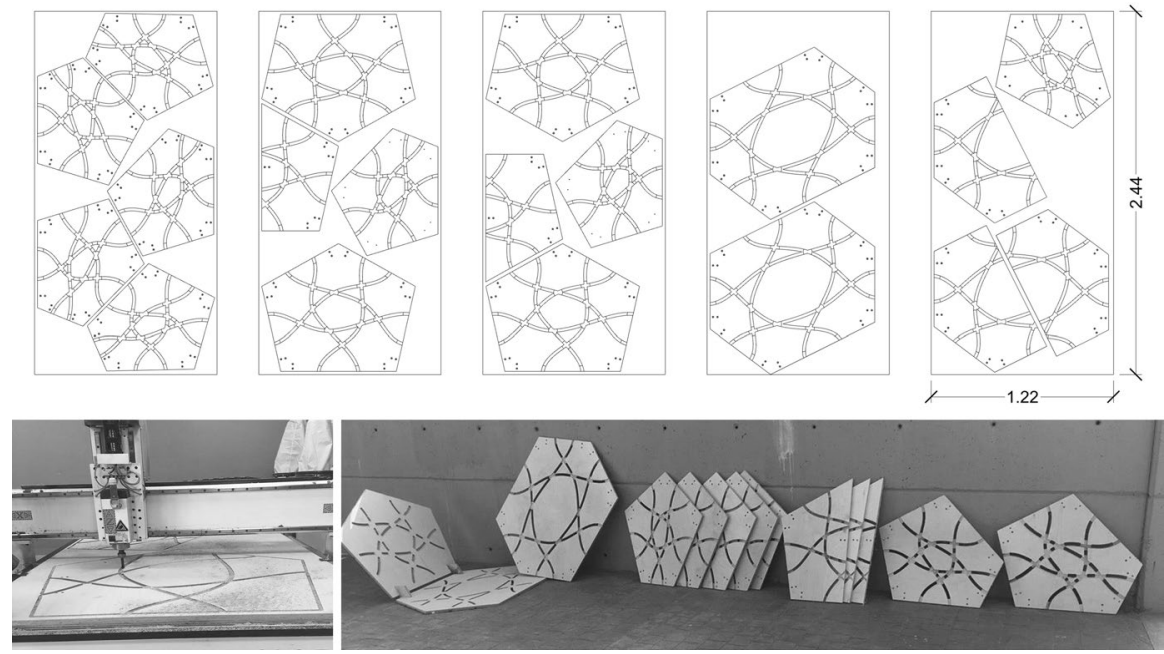

Fig. 8 Drawings and nesting for the three-axis CNC milling machine 
make all the panels. Two other tables were needed for the construction of the joints. The processing time was approximately $1 \mathrm{~h}$ for each table. More complex processes using the robotic arm available at the BAU fabrication laboratory were used in the past. However, the short timeframe available for the construction of the pavilion required simpler and faster solutions. The assembly of the panels that made up each cell was not particularly complex; following a simple connection scheme that joined the cell's panels was sufficient. However, the polyhedral cells of the pavilion had missing faces, and some complications could arise from failure to recognize absent parts and to identify the correct orientation of the panels. However, the structure and its rules soon became familiar and the builders quickly learned how to assemble the parts by observing the digital three-dimensional model.

\section{Conclusion}

The Let's Join pavilion is first stage of an international collaboration at the intersection of research and teaching. This partnership aims to enhance the theory and experimentation of geometric shape in the "ex ante" phase of architectural projects. The use of Weaire and Phelan tessellations to form the structure of the pavilion was successful, confirming the great potential that this multifaceted articulation, which does not involve particular structural and constructive complexities, can offer on an aesthetic and functional level. It should be noted that the pavilion was born as a "host" architectural structure, to allow the design and experimentation of prototypes of responsive dynamic surfaces. Indeed, in its current configuration, the pavilion's polyhedrons are missing some polygonal panels. Some are absent to allow entry and exit from the pavilion. Others, pentagonally-shaped and with different spatial orientations, are intended to house responsive dynamic surfaces (Casale 2020) (Figs. 9, 10). These surfaces will enter into a formal geometric dialogue with the
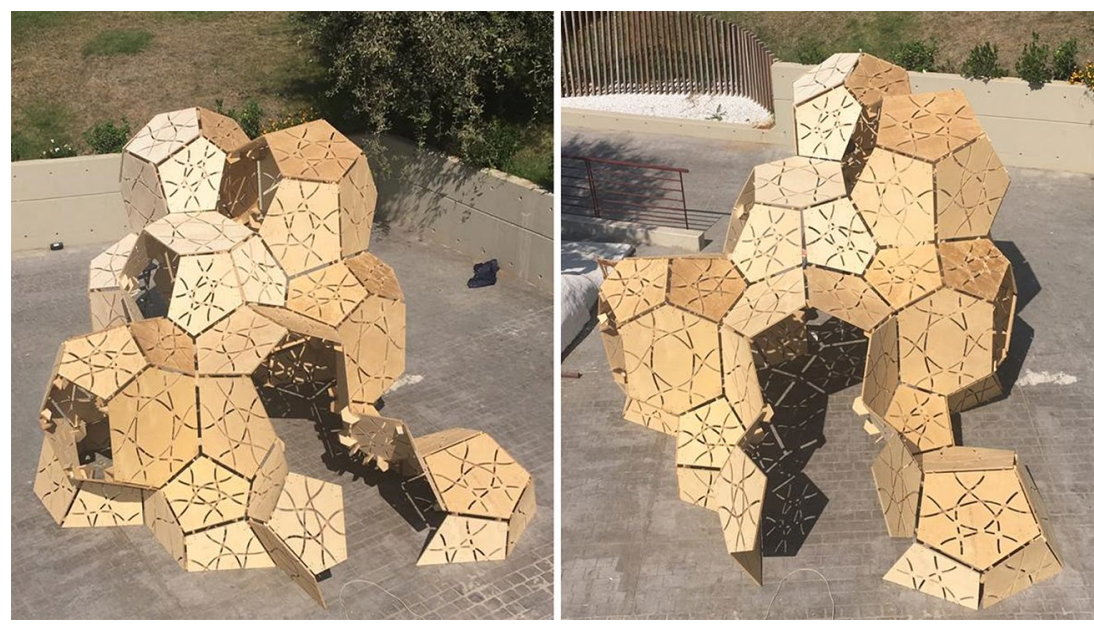

Fig. 9 The pavilion built 


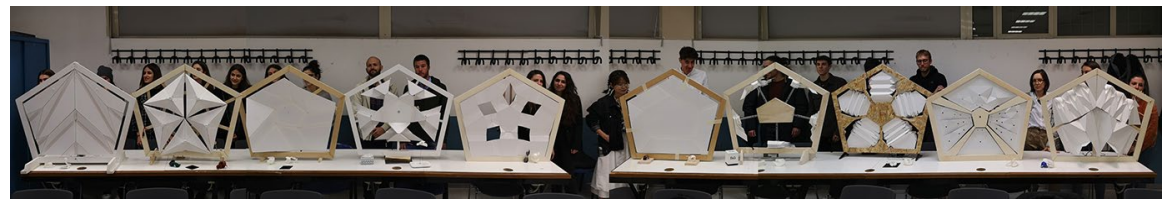

Fig. 10 First design tests of responsive surfaces, carried out by students during the course: "Disegno e modello" of the Degree in Design, at the Sapienza University of Rome

polyhedral tessellation and the pattern of cubic curves placed on it, continuing to enhance the theory and experimentation of geometric form in the "ex ante" phase of the project. In October 2019, shortly after the construction of the pavilion, several didactic and research initiatives had already been launched to elicit proposals for the missing surfaces.

Funding Open Access funding provided by Università degli Studi di Roma La Sapienza.

Open Access This article is licensed under a Creative Commons Attribution 4.0 International License, which permits use, sharing, adaptation, distribution and reproduction in any medium or format, as long as you give appropriate credit to the original author(s) and the source, provide a link to the Creative Commons licence, and indicate if changes were made. The images or other third party material in this article are included in the article's Creative Commons licence, unless indicated otherwise in a credit line to the material. If material is not included in the article's Creative Commons licence and your intended use is not permitted by statutory regulation or exceeds the permitted use, you will need to obtain permission directly from the copyright holder. To view a copy of this licence, visit http://creativeco mmons.org/licenses/by/4.0/.

\section{References}

Alvarez, Martín; Wagner, Hans Jakob; Groenewolt, Abel; Krieg, Oliver David; Kyjanek, Ondrej; Sonntag, Daniel; Bechert, Simon; Aldinger, Lotte; Menges, Achim; Knippers, Jan 2019. The Buga Wood Pavilion. In: Ubiquity and Autonomy-39th ACADIA conference 2019. Austin, USA: Acadia Publishing Company. 490-499.

Baglioni, Leonardo. 2009. I Poliedri Regolari e Semiregolari con un Approfondimento sulle Cupole Geodetiche. In Geometria Descrittiva: Tecniche e Applicazioni. Vol II. Novara: CittàStudi

Baraona Pohn, Ethel. 2008. Watercube: the book. Barcelona: Actar.

Casale, Andrea, Valenti, Graziano Mario and Michele, Calvano. 2020. The shape of the folded surfaces Milano: Franco Angeli Editore

D’Acunto, Pierluigi, and Toni Kotnik. 2013. AA/ETH-Pavilion. In Proceedings of the TENSINET Symposium. Istanbul, Turkey: VUB - Faculty of Physical Education and Physiotherapy. 99-108

Hartmann, Manuel Fabian; Johannes, Stadelmann; Paul, Koeb; Martin, Grießer; Michael, Bachlechner, 2013. Pavillon in Alberschwende https://www.karamba3d.com/project/small -wooden-building/

Hege, Hans-Cristian and Konrad Polthier,. 2012. Visualization and Mathematics: Experiments, Simulations and Environments. Berlin: Springer Science \& Business Media.

Oliver David Krieg, Zachary Christian, David Correa, Achim Menges, Steffen Reichert, Katja Rinderspacher, Tobias Schwinn 2013. HygroSkin: Meteorosensitive Pavilion. In Fabricate: Negotiating Design and Making. Zürich: E T H Honggerberg. 272-279.

Oliver David Krieg, Tobias Schwinn, Achim Menges, Jian-Min Li, Jan Knippers, Annette Schmitt, Volker Schwieger 2015. Biomimetic Lightweight Timber Plate Shells: Computational 
Integration of Robotic Fabrication, Architectural Geometry and Structural Design. In Advances in Architectural Geometry 2014 Springer. 109-125.

Laccone, Francesco, Francesco Laccone, Luigi Malomo, Jesús Pérez, Nico Pietroni, Federico Ponchio, Bernd Bickel, Paolo Cignoni 2019. FlexMaps Pavilion: A Twisted Arc made of Mesostructured Flat Flexible Panels. Proceedings of IASS Annual Symposia. 498-504

Luigi Malomo, Jesús, Emmanuel, Nico Pietroni, Eder, Paolo Cignoni, Bernd 2018. FlexMaps: Computational Design of Flat Flexible Shells for Shaping 3D Objects. ACM Transactions on Graphics Vol. 37 N. 6. Asia: Siggraph. 1-14.

Malone, Meredith, and Igor Marjanovic. 2014. Thomas Saraceno: Cloud-specific. St. Louis, USA: Mildred Lane Kemper Art Museum.

Pohl, Göran. 2011. BOWOOSS: Bionic Optimized Wood Shells with Sustainability. In Proceedings of SPIE - The International Society for Optical Engineering 2011. DOI: 0.1117/12.881778

Schwinn, Tobias, Oliver David, Krieg, and Achim Menges. 2016. Robotic Sewing: A Textile Approach Towards the Computational Design and Fabrication of Lightweight Timber Shells. In Advances in Architectural Geometry 2016. Zurich: AAG.

Schwinn, Tobias; Krieg, Oliver David; Menges, Achim; Mihaylov, Boyan; Reichert, Steffen 2012. Machinic Morphospaces: Biomimetic Design Strategies for the Computational Exploration of Robot Constraint Spaces for Wood Fabrication. In Proceedings of the 32nd Annual Conference of the Association for Computer Aided Design in Architecture (ACADIA). San Francisco. 157-168.

Weaire Denis, Kelvin Problem: Foam Structures of Minimal Surface Area. London: Taylor \& Francis

Publisher's Note Springer Nature remains neutral with regard to jurisdictional claims in published maps and institutional affiliations.

Graziano Mario Valenti is Associate Professor in the Department of History, representation, and restoration of architecture at the University of Rome "La Sapienza". His research activities - divided into theoretical, design, implementation and testing of experimental models - is focused on the application of new digital technologies to the disciplines of the survey, drawing and visual communication, with the aim of anticipating future operating scenarios and solve today application problems. Subject of special study, which crosses all his research, is the definition and representation of an integrated and dynamic model that consists by heterogeneous information.

Chadi El Khoury is an Associate Professor in the Faculty of Architecture - Design and Built Environment, Beirut Arab University since 2016. He studied architecture in Beirut and Rome. He graduated from "Sapienza" University of Rome with supervisor Laura De Carlo. In 2006 he began practice as a professional designer. In 2015 he received his Ph.D. in architectural design from "Sapienza" University of Rome with supervisor Antonino Saggio with the thesis: "Folding". A morpho-semantic model to support the creative phase of the project. He began his teaching experience as an assistant to undergraduate courses at "Sapienza" University of Rome Faculty of Architecture while continuing the practice of the profession of designer. His professional interests include using varied architectural subjects such as architectural design, parametric architecture, and digital fabrication. 